\title{
INEQUALITIES ASSOCIATED WITH REGULAR AND SINGULAR PROBLEMS IN THE CALCULUS OF VARIATIONS
}

\section{J. S. BRADLEY AND W. N. EVERITT}

ABSTRACT. An inequality of the form $\int_{a}^{b}\left[p\left|f^{\prime}\right|^{2}+q|f|^{2}\right] \geq \mu_{0} \int_{a}^{b}|f|^{2}(f \in D)$ is established, where $p$ and $q$ are real-valued coefficient functions and $f$ is a complex-valued function in a set $D$ so chosen that both sides of the inequality are finite. The interval of integration is of the form $-\infty<a<b \leq \infty$. The inequality is first established for functions in the domain of an operator in the Hilbert function space $L^{2}(a, b)$ that is associated with the differential equation $-\left(p y^{\prime}\right)^{\prime}+q y=\lambda y$, and the number $\mu_{0}$ in the inequality is the smallest number in the spectrum of this operator. An approximation theorem is given that allows the inequality to be established for the larger set of functions $D$. An extension of some classical results from the calculus of variations and some spectral theory is then used to give necessary and sufficient conditions for equality and to show that the constant $\mu_{0}$ is best possible. Certain consequences of these conclusions are also discussed.

1. Introduction. We are concerned with inequalities of the form

$$
\int_{a}^{b}\left[p\left|f^{\prime}\right|^{2}+q|f|^{2}\right] \geq \mu_{0} \int_{a}^{b}|f|^{2} \quad(f \in D),
$$

where $p$ and $q$ are real-valued coefficients, with $p(x)>0, \mu_{0}$ is a real number, and $f$ is a complex-valued function in a set $D$ so chosen that both sides of (1.1) are finite. The interval of integration is of the form $-\infty<a<b \leq \infty$; when $b<\infty$ we shall say the problem is regular and when $b=\infty$ that the problem is singular.

Inequalities of the form (1.1) arise in the calculus of variations from the problem of minimizing a functional $\int_{a}^{b}\left[p\left|f^{\prime}\right|^{2}+q|f|^{2}\right]$ in certain classes of functions which also satisfy the normalization condition $\int_{a}^{b}|f|^{2}=1$. The Euler equation for such a problem is

$$
M[y]=\lambda y,
$$

where $\lambda$ is a parameter and $M[$.$] is the second-order linear differential expres-$ sion given by

Presented to the Society, November 26, 1972; received by the editors August 11, 1972 and, in revised from, October 26, 1972.

AMS (MOS) subject classifications (1970). Primary 26A86, 49B10; Secondary 34B25, 47E05. 


$$
M[y]=-\left(p y^{\prime}\right)^{\prime}+q y \text { on }[a, b)
$$

Inequalities of the form (1.1) are sometimes established for functions satisfying certain boundary conditions, as in the work of Putnam [15]; however, we shall be concerned with the free end point problem in that we impose no boundary conditions on the functions for which (1.1) is proved. For a discussion of the relation between calculus of variations and equation (1.2) the reader is referred to the book by Bliss [2], or that by Weinstock [18]; for a discussion of free end point problems see Bliss [2] or Akhiezer [1].

We shall make considerable use of the Dirichlet formula for the differential expression $M$

$$
\int_{a}^{b}\left[p\left|f^{\prime}\right|^{2}+q|f|^{2}\right]=\left[p \bar{f} f^{\prime}\right]_{a}^{b}+\int_{a}^{b} \bar{f} M[f]
$$

and while this result always holds in the regular case it may or may not hold in the singular case. In the latter case we quote results from [6], [7] and [8] which enable us to make use of (1.4) in the singular case also.

The spectral theory of equation (1.2) plays an important part in these problems and, indeed, the real number $\mu_{0}$ in (1.1) is determined by the requirement that it should be the minimum point in the spectrum of certain differential operators generated by $(1.3)$ in the Hilbert function space $L^{2}(a, b)$. This was recognized early in the development of the theory of the calculus of variations; see in particular the memoir by Lichtenstein [13] and the classic account to be found in the Methoden of Courant and Hilbert [5], especially Volume I, Chapter 6. However, these results are mostly concerned with the regular case, although some discussion of special singular problems is found in Chapter $6, \$ \$ 2.5,5$ and 7.10 of Courant and Hilbert.

In the singular case the outstanding contribution was made by Putnam; see [15]. Some of our results have a connection with the conclusions reached by Putnam although we work with a more general differential expression and our results are not contained in the theorems of [15]. We shall make reference to the results in [15] elsewhere in this paper.

In addition to these important references, mention should be made of the chapter on the calculus of variations in the book Inequalities by Hardy, Littlewood, and Pólya [11]. There is an illuminating discussion on the role of the calculus of variations in proving integrodifferential inequalities given in $\$ \$ 7.1$ and 7.2 of that work; however, the discussion is restricted to particular inequalities and where inequalities of the form (1.1) appear the spectral theory of the problem is not specifically considered in the analysis. 
We have stated in Theorem 1 a result for the regular case under general conditions on the coefficients $p$ and $q$. While the regular problem is classic (see for example Chapter V of the text by Reid [16]) we have not been able to find any definite reference to this result in the literature, particularly to the case where $q$ need not be bounded below. However, the proof of this result is subsumed into that for the singular case except for a discussion of one or two points of special interest.

We outline the contents of the paper. In the next section we give the conditions to be satisfied by the coefficients $p$ and $q$ in (1.3), define the set $D$ for which inequality (1.1) will be valid and then state two the orems to cover results in the regular and singular cases. $\$ 3$ contains an approximation theorem needed in the proof of Theorem 2 , and $\$ 4$ contains some variational results. The proof of Theorem 2 is then given in $\$ 5$, and $\$ 6$ contains some observations on modifications needed in the proof of Theorem 1 as well as some consequences of this theorem relating to the ordering of eigenvalues of various operators defined by equation (1.2).

Acknowledgement. J. S. Bradley wishes to thank the University of Dundee for some financial support during the academic year 1971-1972 spent in Dundee. The authors also wish to thank Professor W. T. Reid for his assistance in pointing out some related work in the literature.

2. Statement of results. In the regular case we shall be concerned with the compact interval $[a, b]$ and in the singular case with the half-line $[a, \infty)$.

If $I$ is any interval of the real line $R$ then $L_{1 \propto c}^{2}(l)$ denotes the collection of all complex-valued, Lebesgue measurable functions on $I$ which are integrablesquare on every compact subinterval of $I$. Also, $A C_{\text {loc }}(I)$ represents the collection of all complex-valued functions on $I$ which are absolutely continuous on all compact subintervals of $l$.

The basic conditions required on the real-valued coefficients $p$ and $q$ are:

(1) Regular case.

(2.1) (i) $p$ is absolutely continuous on $[a, b], p^{\prime} \in L^{2}(a, b)$ and $p(x)>0$ on $[a, b]$, (ii) $q \in L^{2}(a, b)$.

(2) Singular case.

(2.2) (i) $p$ is in $A C_{\mathrm{loc}}[a, \infty), p^{\prime}$ is in $L_{1 \infty}^{2}[a, \infty), p(x)>0$ on $[a, \infty)$, (ii) $q$ is in $L_{1 \propto c}^{2}[a, \infty)$.

The reason for requiring $p^{\prime}$ and $q$ to be integrable-square (locally in the singular case) is commented upon in the proof of Theorem 3 below.

We define the following linear manifolds of the Hilbert function spaces $L^{2}(a, b)$ or $L^{2}(a, \infty)$ (recall that $M$ is the differential expression defined by (1.3)): 
(1) Regular case.

(i) $f \in \Delta \equiv \Delta(p, q)$ if $f^{\prime}$ is absolutely continuous on $[a, b]$ and $M[f] \epsilon$ $L^{2}(a, b)$ (note that $\left.\Delta \subset L^{2}(a, b)\right)$,

(ii) $f \in \mathscr{D}(\alpha, \beta) \subset \Delta$, where $\alpha, \beta \in[0, \pi)$, if $f \in \Delta$ and $f(a) \cos \alpha+f^{\prime}(a)$ - $\sin \alpha=0=f(b) \cos \beta+f^{\prime}(b) \sin \beta$,

(iii) $f \in D \equiv D(p, q)$ if $f$ is absolutely continuous on $[a, b]$ and $f^{\prime} \in L^{2}(a, b)$ (note that $f \in D$ implies that $p^{1 / 2} f^{\prime},|q|^{1 / 2} f \in L^{2}(a, b)$ ).

(2) Singular case.

(i) $f \in \Delta \equiv \Delta(p, q)$ if $f \in L^{2}(a, \infty), f^{\prime}$ is in $A C_{\mathrm{loc}}[a, \infty)$ and $M[f] \in L^{2}(a, \infty)$,

(ii) $f \in \mathscr{D}(\alpha) \subset \Delta$, where $\alpha \in[0, \pi)$ if $f \in \Delta$ and $f(a) \cos \alpha+f^{\prime}(a) \sin \alpha=0$, $L^{2}(a, \infty)$.

(iii) $f \in D \equiv D(p, q)$ if $f \in L^{2}(a, \infty), f$ is in $A C_{\mathrm{loc}}[a, \infty), p^{1 / 2} f^{\prime},|q|^{1 / 2} f \epsilon$

Finally, we define the following linear, unbounded differential operators in $L^{2}(a, b)$ or $L^{2}(a, \infty)$ :

(1) Regular case. For each $\alpha, \beta \in[0, \pi)$ define $T(\alpha, \beta): \mathscr{D}(\alpha, \beta) \rightarrow L^{2}(a, b)$ by $T(\alpha, \beta) f=M[f], f \in \mathscr{D}(\alpha, \beta)$.

(2) Singular case. For each $\alpha \in[0, \pi)$ define $T(\alpha): \mathscr{D}(\alpha) \rightarrow L^{2}(a, \infty)$ by $T(\alpha) f$ $=M[f], f \in \mathscr{D}(\alpha)$.

The differential operator $T(\alpha, \beta)$ is selfadjoint in $L^{2}(a, b)$ and has a discrete, simple spectrum. For this result see Naimark $[14, \$ 19.4]$. It is also the case that $T(\alpha, \beta)$ is bounded below in $L^{2}(a, b)$ eventhough when (ii) of (2.1) is assumed $q$ need not be bounded below on $[a, b]$; essentially, this result may be drawn from the book by Glazman $[10, \$ 27$, Theorems 2 and 3]. For an alternative proof see the theorem in [9] where an estimate of the lower bound of $T(\alpha, \beta)$ is given in terms of the norm of $q$. Both of these proofs are given for the singular case but may be adapted for the regular case. If now we denote by $\lambda_{0}(\alpha, \beta)$ the smallest eigenvalue of $T(\alpha, \beta)$ we obtain the inequality

$$
(T(\alpha, \beta) f, f) \geq \lambda_{0}(\alpha, \beta)(f, f) \quad(f \in \mathscr{D}(\alpha, \beta)),
$$

where $(.,$.$) is the inner product in L^{2}(a, b)$. For a proof of this inequality see the book by Glazman $[10$, p. 15].

In the singular case we need additional conditions on the coefficients $p$ and $q$ in order to secure $T(\alpha)$ as a selfadjoint operator with a spectrum that is bounded below. We shall discuss the problem under two distinct conditions as follows:

(2.4)(a) The bound condition. There is a real number $d$ such that $q(x)$ $\geq d(x \in[a, \infty))$ (no additional conditions on $p$ ).

(2.5)(b) The integrable condition. $p(x)=1(x \in[a, \infty))$ and for some $r \geq 1, q \in L^{r}(a, \infty)$. 
We note first of all that both (2.4) and (2.5) are sufficient to put the differential expression $M$ in the limit-point case at $\infty$; for (2.4) see [6] and then $[7, \S 3]$; for (2.5) see Theorem 2 of [8]. (Note that in the integrable condition (2.5) $q$ need not be bounded below.) It then follows in both cases that $T(\alpha)$ is selfadjoint in $L^{2}(a, \infty)$; see Naimark $[14, \S 18.3]$.

It also follows in the singular case that (2.4) or (2.5) is sufficient to ensure that $T(a)$ is bounded below in $L^{2}(a, \infty)$; for (2.4) see Theorem 1 in $\$ 38$ in the book by Glazman [10], for (2.5) see Theorem 2 and 3 of $\$ 27$ of the same book, or an alternative proof in [9]. As in the regular case, if we now define $\lambda_{0}(\alpha)$ to be the smallest number in the spectrum of $T(\alpha)$, we have the inequality

$$
(T(\alpha) f, f) \geq \lambda_{0}(\alpha)(f, f) \quad(f \in \mathcal{D}(a)) .
$$

We introduce here for use below a notation for the spectrum of a selfadjoint operator $T$ in $L^{2}$, where we follow the account in $\$ 4$ of [4]. The resolvent set of $T$ is $\rho(T)$; the spectrum $\sigma(T)$, which is a closed set of the real line $R$, is written as the union of three disjoint sets $\sigma(T)=\operatorname{Po}(T) \cup \operatorname{Co}(T) \cup \operatorname{PC} \sigma(T)$, respectively the point, continuous, and point-continuous spectra of $T . \mathrm{P} \sigma(T)$ is the set of isolated eigenvalues of $T$ of finite multiplicity, $\mathrm{PC}(T)$ is the set eigenvalues embedded in the continuous spectrum $C \sigma(T)$ or isolated eigenvalues of infinite multiplicity. The latter type of eigenvalues cannot occur in the problems considered here.

It is convenient at this stage to mention the Dirichlet formula. For the regular case this will take the form, on an integration by parts,

$$
\int_{a}^{b}\left[p f^{\prime} \bar{g}^{\prime}+q \bar{g}\right]=\left[p f^{\prime} \bar{g}\right]_{a}^{b}+\int_{a}^{b} \bar{g} M[f] \quad(g \in D, f \in \Delta) .
$$

In the singular case the corresponding result is more difficult to prove and depends on the following results which we state as

Lemma 1. Let $p$ and $q$ satisfy (2.2) and either (2.4) or (2.5); let $D$ and $\Delta$ be defined as above. Then

(a) in the bound condition $p^{1 / 2} f^{\prime}$ and $|q|^{1 / 2} f \in L^{2}(a, \infty)$ for all $f \in \Delta$ and

$$
\lim _{x \rightarrow \infty} p(x) f^{\prime}(x) \bar{g}(x)=0 \quad(g \in D, f \in \Delta) ;
$$

(b) in the integrable condition $f^{\prime}$ and $|q|^{1 / 2} f \in L^{2}(a, \infty)$ for all $f$ in $\Delta$ and

$$
\lim _{x \rightarrow \infty} f^{\prime}(x) \bar{g}(x)=0 \quad(g \in D, f \in \Delta) .
$$

The proof of (2.8) will be found in [6] and then in $\$ 3$ of [7]; it is shown there that (2.8) holds for all $f, g \in \Delta$ but an examination of the proof shows that it 
extends if this condition is relaxed to $f \in \Delta, g \in D$. The proof of (2.9) is found in [9, Theorem 2], again for $f, g \in \Delta$ but the extension to $f \in \Delta, g \in D$ also follows from the analysis given there.

With these results we may state the Dirichlet formula in the singular case:

(a) in the bound condition

$$
\int_{a}^{\infty}\left[p f^{\prime} \bar{g}^{\prime}+q \bar{f} \bar{g}\right]=-p(a) f^{\prime}(a) \bar{g}(a)+\int_{a}^{\infty} \bar{g} M[f] \quad(g \in D, f \in \Delta) ;
$$

(b) in the integrable condition

$$
\int_{a}^{\infty}\left[f^{\prime} \bar{g}^{\prime}+q f \bar{g}\right]=-f^{\prime}(a) \bar{g}(a)+\int_{a}^{\infty} \bar{g} M[f] \quad(g \in D, f \in \Delta) .
$$

All three separate integrals involved in these formulae are absolutely convergent.

We are now in a position to state the results to be proved in this paper. Firstly,

Theorem 1 (The regular case). Let the coefficients $p$ and $q$ satisfy the conditions (2.1) and let the linear manifold $D$ of $L^{2}(a, b)$ be defined as above. Then the following inequality is valid:

$$
\int_{a}^{b}\left[p\left|f^{\prime}\right|^{2}+q|f|^{2}\right] \geq \mu_{0} \int_{a}^{b}|f|^{2} \quad(f \in D)
$$

where the real number $\mu_{0}$ is defined by

$$
\mu_{0}=\inf \sigma(T(\pi / 2, \pi / 2)) ;
$$

that is, $\mu_{0}$ is the smallest eigenvalue of the operator $T(\pi / 2, \pi / 2)$, which is bounded below in $L^{2}(a, b)$.

There is equality in (2.11) if and only if for some complex number $c$ we bave $f=c \psi_{0}$, where $\psi_{0}$ is an eigenfunction of $T(\pi / 2, \pi / 2)$ corresponding to $\mu_{0}$

Secondly,

Theorem 2 (The singular case). Let the coefficients $p$ and $q$ satisfy the conditions (2.2) and (2.4) or (2.5) and let the linear manifold $D$ of $L^{2}(a, \infty)$ be defined as above. Then the following inequalities are valid:

(a) the bound condition

$$
\int_{a}^{\infty}\left[p\left|f .^{\prime}\right|^{2}+q|f|^{2}\right] \geq \mu_{0} \int_{a}^{\infty}|f|^{2} \quad(f \in D)
$$

(b) the integrable condition

$$
\int_{a}^{\infty}\left[\left|f^{\prime}\right|^{2}+q|f|^{2}\right] \geq \mu_{0} \int_{a}^{\infty}|f|^{2} \quad(f \in D),
$$


where in both cases the real number $\mu_{0}$ is defined by $\mu_{0}=\inf \sigma(T(\pi / 2))$; that is, $\mu_{0}$ is the smallest number in the spectrum of the operator $T(\pi / 2)$, which is bound. ed below in $L^{2}(a, \infty)$.

If $\mu_{0} \in \mathrm{P} \sigma(T(\pi / 2)) \cup \operatorname{PC} \sigma(T(\pi / 2))$, then there is equality in (2.13) or (2.14) if, and only if, there is a complex number $c$ such that $f=c \psi_{0}$, where $\psi_{0}$ is an eigenfunction for $T(\pi / 2)$ corresponding to $\mu_{0}$.

If $\mu_{0} \in \mathrm{C} \sigma(T(\pi / 2))$, then there is equality in (2.13) or (2.14) if, and only if, $f$ is the zero function; however, the inequality is the best possible in the sense that there is a sequence $\left\{f_{n}: n=1,2, \ldots\right\}$ such that $f_{n} \in D, \int_{a}^{\infty}\left|f_{n}\right|^{2}=1 \quad(n=$ $1,2, \ldots)$ and in the bound condition

$$
\lim _{n \rightarrow \infty} \int_{a}^{\infty}\left[p\left|f_{n}^{\prime}\right|^{2}+q\left|f_{n}\right|^{2}\right]=\mu_{0}
$$

with a corresponding result with $p=1$ in the integrable condition.

We now compare Theorems 1 and 2 to the results obtained by Putnam in [15], particularly Theorems I and II of that paper. Both papers deal with an inequality of the form (2.13). Putnam requires that $p(x)=1$, that $q$ be continuous and bounded below on $[0, \infty)$, whereas here we do not require $q$ to be bounded below where $p(x)=1$, and when we require $q$ to be bounded below we allow $p$ to be any positive, locally absolutely continuous function with a locally square-integrable derivative.

Another significant difference between Putnam's results and those obtained here lies in the class of functions considered in each case. Putnam's inequality, as given in his Theorems I and II is established for the class of functions $\Omega_{0}$ that are continuous, have piecewise continuous derivatives, are square-integrable on $[0, \infty)$ and vanish at the origin. Since our Theorem 2 is valid for all $f$ in $D$ and $\Omega_{0} \subset D$, this theorem is more general than Theorem II of [15]. However, we do not have a direct extension because the number $\mu_{0}$ in (2.13) is replaced by a larger number $\lambda_{0}$ in Putnam's version so that his inequality is sharper on the smaller set $\Omega_{0}$. This last remark raises the interesting question as to whether (2.13) remains true for functions $f$ in $D$ with $f(0)=0$ if one replaces $\mu_{0}$ by $\min \{\lambda: \lambda \in \sigma(T(0))\}$.

We should also remark that there is a more direct comparison between Theorem I (bis) of Putnam and our Theorem 2. Here Putnam's result is valid for a set $\bigcup_{a \epsilon[0, \pi)} \Omega_{a}$ which is strictly contained in our set $D$ but his lower bound is connected with the spectrum of $T(\pi / 2)$ as in Theorem 2; however, in this case Putnam does not give any discussion of cases of equality.

Finally, we observe that it is enough to establish Theorem 1 and 2 for real- 
valued functions in $D$ since the real-valued components of any complex-valued function in $D$ are themselves in $D$. Therefore, we consider only real-valued functions in $D$ for the remainder of this paper.

3. An approximation theorem. Our proof of Theorem 2 requires the following approximation results. In view of the remarks at the end of $\$ 2$, we assume that all functions in the sequel are real-valued.

Theorem 3. Suppose that the coefficients $p$ and $q$ satisfy conditions (2.2) and (2.4) or (2.5). Given $f$ in $D$ and $\epsilon>0$, there is a function $g$ in $\mathscr{T}(\pi / 2)$ baving compact support and the property that

$$
\left|\int_{a}^{\infty} p f^{\prime 2}-\int_{a}^{\infty} p g^{\prime 2}\right|<\epsilon,\left|\int_{a}^{\infty} f^{2}-\int_{a}^{\infty} g^{2}\right|<\epsilon, \quad\left|\int_{a}^{\infty} q f^{2}-\int_{a}^{\infty} q g^{2}\right|<\epsilon .
$$

We use the following lemma in the proof of Theorem 3.

Lemma 2. If the coefficients $p$ and $q$ satisfy conditions (2.2) and (2.4) or (2.5), $f$ is a function in $D$, and $\epsilon_{1}>0$, then there is a number $X>a$ with the property that

$$
\int_{x}^{\infty} p f^{\prime 2}<\epsilon_{1}, \quad \int_{x}^{\infty}|q| f^{2}<\epsilon_{1}, \quad \int_{x}^{\infty} f^{2}<\epsilon_{1},
$$

and

$$
|f(X)|^{2} \int_{a}^{X}|q|<\epsilon_{1}, \quad(X-a)|f(X)|^{2}<\epsilon_{1} .
$$

Proof. The fact that (3.1) holds for all sufficiently large $X$ follows from the assumption that $f \in D$. The proof of (3.2) depends on whether (2.4) or (2.5) holds. Suppose that (2.4) holds. Then in view of the inequality (1.1) in question, there is no loss in generality in assuming that $q(t) \geq k>0(t \in[a, \infty))$. If follows that there is a sequence $\left\{x_{n}\right\}, x_{n} \rightarrow \infty$, with the property that $\left|f\left(x_{n}\right)\right|^{2} \int_{a}^{x_{n}} q \rightarrow$ 0 as $n \rightarrow \infty$. If not, there is a number $c>a$ and a number $m>0$ such that $|f(x)|^{2} \geq m / \int_{a}^{x} q$ for all $x \geq c$. Multiplication by $q$ and integration yields

$$
\int_{c}^{x}|f(t)|^{2} q(t) d t \geq m \int_{c}^{x}\left(q(t) / \int_{a}^{t} q(s) d s\right) d t=m\left[\log \int_{a}^{x} q-\log \int_{a}^{c} q\right] .
$$

Since $q(t) \geq k>0$, the right-hand side of this last inequality tends to infinity with $x$ and this is a contradiction of the assumption that $f \in D$. Therefore the desired sequence exists. Now $\int_{a}^{x_{n}} q \geq \int_{a}^{x_{n}} k=k\left(x_{n}-a\right)$, and $\left|f\left(x_{n}\right)\right|^{2} \int_{a}^{x_{n}} q \geq$ $k\left(x_{n}-a\right)\left|f\left(x_{n}\right)\right|^{2}$, from which it follows that $\left(x_{n}-a\right)\left|f\left(x_{n}\right)\right|^{2} \rightarrow 0$. It is now clear that this lemma is valid in case (2.4) holds. 
We now suppose that (2.5) holds, and note that there is a sequence $\left\{x_{n}\right\}$, $x_{n} \rightarrow \infty$, with the property that $\left(x_{n}-a\right)\left|f\left(x_{n}\right)\right|^{2} \rightarrow 0$, since otherwise there is a constant $c>0$ with $|f(x)|^{2} \geq c /(x-a)$, for all sufficiently large $x$, and this is inconsistent with $f \in L^{2}$. It follows from condition (2.5), with $r>1,1 / r+1 / s=1$, $K=\left(\int_{a}^{\infty}|q|^{r}\right)^{1 / r}$, that

$$
\int_{a}^{x_{n}}|q| \leq\left(\int_{a}^{x_{n}}|q|^{r}\right)^{1 / r}\left(\int_{a}^{x_{n}} 1\right)^{1 / s} \leq K\left(x_{n}-a\right)^{1 / s} \leq K\left(x_{n}-a\right)
$$

for all sufficiently large $n$. If $r=1$ in (2.5), the inequality

$$
\left.\int_{a}^{x_{n}}|q| \leq K\left(x_{n}-a\right) \quad \text { (all sufficiently large } n\right)
$$

is immediate. After multiplying both sides of (3.3) by $\left|f\left(x_{n}\right)\right|^{2}$ it is clear that $\left|f\left(x_{n}\right)\right|^{2} \int_{a}^{x_{n}}|q| \rightarrow 0$ and the lemma follows.

Proof of Theorem 3. For $\epsilon_{1}>0$ we choose $X>a$ as in Lemma 1, and then for a positive number $\eta$, we can choose a continuously differentiable function $\phi$ with the property that $\phi(a)=0=\phi(X)=\phi^{\prime}(a)=\phi^{\prime}(X), \phi(x)=0$ on $[X, \infty)$, and $\int_{a}^{X}\left|f^{\prime}-\phi\right|^{2}<\eta$. The existence of $\phi$ follows from the facts that $f \in D$ and that the set of continuously differentiable functions vanishing with their derivatives at $a$ and at $X$ is dense in $L^{2}(a, X)$. Next we define a function $g$ by

$$
\begin{array}{ll}
g(x)=-\int_{x}^{X} \phi & (x \in[a, X]), \\
g(x)=0 & (x \geq X) .
\end{array}
$$

We make one critical remark at this point. A direct observation shows that $g \in \Delta$ since $g$ has a continuous second derivative on $[a, \infty)$, vanishes outside $[a, X]$ and $p, p^{\prime}$ and $q$ are all in $L^{2}(a, X)$ in view of the local integrable-square condition (2.2) on these coefficients. It is at this stage that the condition (2.2) on $p$ and $q$ is essential to our argument. We have not been able to decide if Theorem 2 remains valid when the condition (2.2) is relaxed to requiring only that $p^{\prime}$ and $q$ are locally integrable on $[a, \infty)$.

We have $g \in \Delta, g^{\prime}=\phi, g^{\prime}(0)=\phi(0)=g^{\prime}(X)=\phi(X)=0=g(X)$ and

$$
\int_{a}^{\infty} p\left|f^{\prime}-g^{\prime}\right|^{2}=\int_{a}^{X} p\left|f^{\prime}-g^{\prime}\right|^{2}+\int_{X}^{\infty} p f^{\prime 2} \leq P(X) \eta+\epsilon_{1},
$$

where $P(X)=\max \{p(x): x \in[a, X]\}$.

Next we note that $-(f(a)-g(a))=-(f(X)-g(X))+\int_{a}^{X} f^{\prime}-g^{\prime}$ and $\mid f(a)-$ $g(a)|\leq| f(X)-g(X)|+| \int_{a}^{X} f^{\prime}-g^{\prime}|\leq| f(X)\left|+\int_{a}^{X}\right| f^{\prime}-\phi \mid$. Using Cauchy-Schwarz on the right side we obtain 


$$
|f(a)-g(a)| \leq|f(X)|+(X-a)^{1 / 2}\left(\int_{a}^{X}\left|f^{\prime}-\phi\right|^{2}\right)^{1 / 2},
$$

and it follows that

$$
|f(a)-g(a)|^{2} \leq 2|f(X)|^{2}+2(X-a)\left(\int_{a}^{X}\left|f^{\prime}-\phi\right|^{2}\right) .
$$

Moreover, for any $x \in[a, X]$ we have $g(x)-f(x)=g(a)-f(a)+\int_{a}^{X}\left[f^{\prime}-g^{\prime}\right]$, and $|g(x)-f(x)| \leq|g(a)-f(a)|+\int_{a}^{X}\left|f^{\prime}-\phi\right| \leq|g(a)-f(a)|+(X-a)^{1 / 2}\left(\int_{a}^{X}\left|f^{\prime}-\phi\right|^{2}\right)^{1 / 2}$. Squaring and using (3.5) we find that

$$
|g(x)-f(x)|^{2} \leq 4|f(X)|^{2}+6(X-a) \int_{a}^{X}\left|f^{\prime}-\phi\right|^{2},
$$

for all $x$ in $[a, X]$, and an integration yields

$$
\int_{a}^{X}|g-f|^{2} \leq 4(X-a)|f(X)|^{2}+6(X-a)^{2} \int_{a}^{X}\left|f^{\prime}-\phi\right|^{2} .
$$

It now follows from Lemma 2 that

$$
\int_{a}^{X}|g-f|^{2} \leq 4 \epsilon_{1}+6(X-a)^{2} \eta .
$$

Now we multiply both sides of (3.6) by $|q|$, integrate, and apply Lemma 2 to obtain

$$
\int_{a}^{X}|q||g-f|^{2} \leq 4 \epsilon_{1}+6 \eta(X-a) \int_{0}^{X}|q| .
$$

The plan now is to use (3.4), (3.7), and (3.8) to establish the inequalities in the conclusion of the theorem. It will be convenient to use the notation $\|\psi\|^{2}=\int_{a}^{\infty}|\psi|^{2},\|\psi\|_{X}^{2}=\int_{a}^{X}|\psi|^{2}$ for a function $\psi$ in $L^{2}(a, \infty)$. In this notation we have

$$
\begin{aligned}
& \left|\int_{a}^{\infty} f^{2}-\int_{a}^{\infty} g^{2}\right| \leq|\|f\|-\|g\||(\|f\|+\|g\|) \\
& \quad \leq\|f-g\|(\|f\|+\|g\|)=\left(\int_{a}^{x}|f-g|^{2}+\int_{x}^{\infty} f^{2}\right)^{1 / 2}(\|f\|+\|g\|) .
\end{aligned}
$$

But $\|g\|=\|g\|_{X} \leq\|f\|_{X}+\|g-f\|_{X} \leq\|f\|+\|g-f\|_{X}$. Hence it follows from (3.7) and Lemma 2 that

$$
\left|\int_{a}^{\infty} f^{2}-\int_{a}^{\infty} g^{2}\right| \leq\left[5 \epsilon_{1}+6(X-a)^{2} \eta\right]^{1 / 2}\left[2\|f\|+\left(4 \epsilon_{1}+6(X-a)^{2} \eta\right)^{1 / 2}\right]
$$

Similarly, it follows from (3.4) and Lemma 2 that

$$
\left|\int_{a}^{\infty} p f^{\prime 2}-\int_{a}^{\infty} p g^{\prime 2}\right| \leq\left[P(X) \eta+\epsilon_{1}\right]^{1 / 2}\left[2\left\|p^{1 / 2} f^{\prime}\right\|+\left(P(X) \eta+\epsilon_{1}\right)^{1 / 2}\right] .
$$


The last inequality in Theorem 3 involves $q$ and if (2.5) holds, $q$ may take on negative values. This makes the remaining calculations slightly different from those used to obtain (3.9) and (3.10). First we write

$$
\begin{aligned}
& \left|\int_{a}^{\infty} q f^{2}-\int_{a}^{\infty} q g^{2}\right|=\left|\int_{a}^{\infty} q\left(f^{2}-g^{2}\right)\right| \\
& =\left|\int_{a}^{x} q\left(f^{2}-g^{2}\right)+\int_{x}^{\infty} q f^{2}\right| \leq \int_{a}^{x}|q|\left|f^{2}-g^{2}\right|+\int_{x}^{\infty}|q| f^{2} .
\end{aligned}
$$

Next we note that

$$
\begin{aligned}
\int_{a}^{x}|q|\left|f^{2}-g^{2}\right|=\int_{a}^{x}|q||| f|-| g||(|f|+|g|) \\
\leq\left(\int_{a}^{x}|q||f-g|^{2}\right)^{1 / 2}\left(\int_{a}^{x}|q|(|f|+|g|)^{2}\right)^{1 / 2} \\
\quad \leq\left(\int_{a}^{x}|q||f-g|^{2}\right)^{1 / 2}\left(2 \int_{a}^{x}|q|\left(f^{2}+g^{2}\right)\right)^{1 / 2} .
\end{aligned}
$$

But from this last inequality and (3.8) we have

$$
\begin{aligned}
\int_{a}^{X}|q|\left|f^{2}-g^{2}\right| \leq\left(4 \epsilon_{1}+6 \eta(X-a) \int_{a}^{X}|q|\right)^{1 / 2}\left[\sqrt{2}\left(\left\||q|^{1 / 2} f\right\|_{X}+\left\||q|^{1 / 2} g\right\|_{X}\right)^{1 / 2}\right] \\
\quad \leq\left(4 \epsilon_{1}+6 \eta(X-a) \int_{a}^{X}|q|\right)^{1 / 2}\left[\sqrt{2}\left\{2\left\||q|^{1 / 2} f\right\|_{X}+\left(4 \epsilon_{1}+6 \eta(X-a) \int_{a}^{X}|q|\right)^{1 / 2}\right\}\right] .
\end{aligned}
$$

Then it follows from (3.11), (3.12) and Lemma 2 that

$$
\begin{aligned}
& \left|\int_{a}^{\infty} q f^{2}-\int_{a}^{\infty} q g^{2}\right| \\
& \quad \leq \sqrt{2}\left[4 \epsilon_{1}+6 \eta(X-a) \int_{a}^{X}|q|\right]^{1 / 2}\left[2\left\||q|^{1 / 2} f\right\|_{X}+\left(4 \epsilon_{1}+6 \eta(X-a) \int_{a}^{X}|q|\right)^{1 / 2}\right]+\epsilon_{1} .
\end{aligned}
$$

Suppose now that $\epsilon_{2}>0$ given. We can assume that $\epsilon_{2}<1$ and choose $\epsilon_{1}<\epsilon_{2}^{2} / 10$, choose $X$ as in Lemma 2 , and then choose $\eta$ such that $P(X)_{\eta}<$ $\epsilon_{1}, 6 \eta(X-a) \int_{a}^{X}|q|<\epsilon_{1}, 6(X-a)^{2} \eta<\epsilon_{1}$, and choose $\phi$ and $g$ as indicated above. Then it follows from (3.9) that

$$
\left|\int_{a}^{\infty} f^{2}-\int_{a}^{\infty} g^{2}\right| \leq \epsilon_{2}\left[2\|f\|+\epsilon_{2}\right]
$$

from (3.10) that

$$
\left|\int_{a}^{\infty} p f^{\prime 2}-\int_{a}^{\infty} p g^{\prime 2}\right| \leq \epsilon_{2}\left[2\left\|p^{1 / 2} f^{\prime}\right\|+\epsilon_{2}\right]
$$


and from (3.13) that

$$
\left|\int_{a}^{\infty} q f^{2}-\int_{a}^{\infty} q g^{2}\right| \leq \sqrt{2} \epsilon_{2}\left[2\left\||q|^{1 / 2} f\right\|+\epsilon_{2}\right]+\epsilon_{2} .
$$

The validity of Theorem 3 is now evident from the se last three inequalities, since $\epsilon_{2}$ is an arbitrary positive number.

4. Variational results. We present in this section some extensions of classical results from the calculus of variations that will be used in the proof of Theorem 2.

Lemma 3. If $g$ is integrable on a closed interval $[a, b]$ and if for every absolutely continuous function $f$ with the property that $f(a)=0=f(b)$ we have $\int_{a}^{b} f^{\prime} g=0$, then $g(x)$ is equal to a constant almost everywbere on $[a, b]$.

The proof given in the text by Bliss [2] of the fundamental lemma of the calculus of variations is easily extended to this lemma.

We introduce the notation

$$
J(f, g)=\int_{a}^{\infty}\left[f^{\prime} p g^{\prime}+f q g\right],
$$

defined for functions $f, g$ in $D$.

Theorem 4. If $u$ is locally absolutely continuous on $[a, \infty)$ and $J(u, f)=0$ for all functions $f$ with the property that $f$ is locally absolutely continuous, $f(a)=0$ and $f$ bas compact support on $[a, \infty)$, then $M[u](x)=0$ for almost every $x \in[a, \infty)$.

Proof. Choose $X>a$. Then $J(u, f)=0$ for all absolutely continuous functions $f$ on $[a, X]$ for which $f(a)=0=f(X)$. Therefore for any such $f$

$$
\begin{aligned}
0=\int_{a}^{X} u^{\prime} p f^{\prime}+u q f= & \int_{a}^{X} u^{\prime} p f^{\prime}+\left.\left[f(t) \int_{a}^{t} q(s) u(s) d s\right]\right|_{a} ^{x} \\
& -\int_{a}^{X}\left[f^{\prime}(t) \int_{a}^{t} q(s) u(s) d s\right] d t=\int_{a}^{X} f^{\prime}\left[p u^{\prime}-\int_{a}^{t} q u\right] d t,
\end{aligned}
$$

and it follows from Lemma 3 that the function $v$ defined by $v(t)=p(t) u^{\prime}(t)-$ $\int_{a}^{t} q u(t \in[a, X])$ is equal to a constant $c$ almost everywhere on $[a, X]$. Then

$$
u^{\prime}(t)=\frac{v(t)}{p(t)}+\frac{1}{p(t)} \int_{a}^{t} q u \quad(t \in[a, X])
$$

and therefore for every $x \in[a, X]$ we have

$$
u(x)=u(a)+c \int_{a}^{x} \frac{1}{p(t)} d t+\int_{a}^{x} \frac{1}{p(t)}\left(\int_{a}^{t} q(u) u(s) d s\right) d t
$$


since $v(t)=c$ a.e. on $[a, X]$. Differentiation of both sides of this last equation and multiplication by $p(t)$ yields $p(t) u^{\prime}(t)=c+\int_{s}^{t} q(s) u(s) d s$, which is equivalent to $M[u]=0$ on $[a, X]$. But $X$ was any number greater than $a$ and therefore $M[u]$ $=0$ a.e. on $[a, \infty)$.

To simplify notation we let $J(f)=J(f, f)$.

Corollary. If $J(f) \geq 0$ for all $f$ in $D$ and there is a function $u$ in $D$ for which $J(u)=0$, then $M[u]=0$ a.e. on $[a, \infty)$.

Proof. Choose a function $f$ in $D$ and for each real number $a$ let $f_{\alpha}=u+\alpha$. Then $f_{a} \in D$ and

$$
0 \leq J\left(f_{a}\right)=J(u)+2 \alpha J(u, \alpha)+\alpha^{2} J(f)=2 \alpha J(u, f)+\alpha^{2} J(f) .
$$

This inequality can hold for all real $a$ only if $J(u, f)=0$, and this fact and Theorem 4 imply that $M[u]=0$ a.e. on $[a, \infty)$.

We note that the conclusion of the corollary can be obtained under weaker hypotheses, but the corollary as stated is adequate for our purposes.

5. Proof of Theorem 2. We first show that the inequality (1.1) is valid in $\mathscr{D}(\pi / 2)$, and we use Theorem 3 to extend (1.1) to all of $D$. To obtain conditions for equality in (1.1) we shall appeal to Theorem 4 and its corollary.

For $f$ in $\mathscr{D}(\pi / 2)$,

$$
\int_{a}^{\infty}\left[p f^{\prime 2}+q f^{2}\right]=\int_{a}^{\infty} f M[f] \geq \mu_{0} \int_{a}^{\infty} f^{2},
$$

since $T(\pi / 2)$ is bounded below; that is, $(T(\pi / 2) f, f) \geq \mu_{0}(f, f)(f \in \mathcal{T}(\pi / 2))$.

We are ready to prove that $(1.1)$ holds in $D$. If not, there is a function $f$ in $D$ and a number $\delta>0$ such that $\int_{a}^{\infty}\left[p f^{\prime 2}+q f^{2}\right]-\mu_{0} \int_{a}^{\infty} f^{2}=-\delta$. Then we choose $\epsilon<\min \left\{\delta / 3,\left|\mu_{0}\right| \delta / 3\right\}$ and choose $g$ in $\mathscr{D}(\pi / 2)$ as in Theorem 3. Then

$$
\begin{aligned}
& \int_{a}^{\infty}\left[p g^{\prime 2}+\left(q-\mu_{0}\right) g^{2}\right] \\
& \quad \leq\left|\int_{a}^{\infty} p g^{\prime 2}-\int_{a}^{\infty} p f^{\prime 2}\right|+\left|\int_{a}^{\infty} q g^{2}-\int_{a}^{\infty} q f^{2}\right|+\left|\mu_{0} \int_{a}^{\infty} f^{2}-\mu_{0} \int_{a}^{\infty} g^{2}\right|-\delta \\
& \quad<\delta-\delta=0 .
\end{aligned}
$$

But we have already established that (1.1) holds on $\mathcal{D}(\pi / 2)$. This contradiction implies that (1.1) also holds on $D$.

To show that the inequality obtained is best possible, and to consider the case of equality we consider separately the situation when (a) $\mu_{0} \in \operatorname{Po}(T(\pi / 2))$ $\cup \operatorname{PC} \sigma(T(\pi / 2))$ and (b) $\mu_{0} \in \mathrm{C}(T(\pi / 2))$.

When (a) holds, that is, $\mu_{0} \in \operatorname{Po}(T(\pi / 2)) \cup \operatorname{PC} \sigma(T(\pi / 2))$, then it is clear from (2.10) and (2.11) that equality in (1.1) holds when $f=c \psi_{0}$. Conversely, if $f$ is 
a function in $D$ for which equality holds in (1.1), then a direct application of the corollary of Theorem 4, with $q$ replaced by $q-\mu_{0}$ in (4.1) and (1.3), yields the conclusion that $-(p f)^{\prime}+q f=\mu_{0} f$. We have already noted that this differential equation is in the limit point case, and therefore any $L^{2}$ solution must be a multiple of $\psi_{0}$. But by definition of $D, f$ is in $L^{2}(a, \infty)$ and therefore $f$ is a constant multiple of $\psi_{0}$.

We consider now the case when (b) holds; i.e. $\mu_{0} \in \operatorname{Co}(T(\pi / 2))$. To show that $\mu_{0}$ is the best possible constant it is sufficient to show that $(2.15)$ is satisfied. From the analysis in $\$ 14.9$ of Naimark [14], we obtain the existence of a sequence $\left\{f_{n}: n=1,2, \ldots\right\}$ satisfying the conditions that $f_{n} \in \mathcal{D}(T(\pi / 2)), \int_{a}^{\infty}|f|^{2}=1 \quad(n=$ $1,2, \ldots)$, and $T(\pi / 2) f_{n}-\mu_{0} f_{n} \rightarrow 0$ in $L^{2}$ as $n \rightarrow \infty$. This last condition implies that $\left(T(\pi / 2) f_{n}-\mu_{0} f_{n}, f_{n}\right) \rightarrow 0$ from which it is clear that this sequence satisfies (2.15).

To show that equality can occur only when $f$ is the zero function, suppose to the contrary that there is an $f \neq 0$ for which equality holds in (2.13). Then it follows from the corollary to Theorem 4 , again with $q$ replaced by $q-\mu$ in (4.1) and (1.3), that $f \in \Delta$ and $\left(M-\mu_{0}\right)[f]=0$ on $[a, \infty)$. An application of the Dirichlet formula $(2.10 \mathrm{a})$ or $(2.10 \mathrm{~b})$ shows that $f(a) f^{\prime}(a)=0$. Since $\mu_{0} \in \operatorname{Co}(T(\pi / 2)), \mu_{0}$ is not an eigenvalue of $T(\pi / 2)$ and therefore $f^{\prime}(a) \neq 0$. Therefore $f(a)=0$ and $\mu_{0}$ is an eigenvalue of $T(0)$. To show that this is impossible we invoke the connection between the spectrum of the operators $T(0), T(\pi / 2)$ and the analytic properties of the Weyl $m$-coefficients $m(\lambda, 0)$ and $m(\lambda, \pi / 2)$, where $\lambda$ is the complex spectral parameter in (1.2); for a discussion of this connection see $[4, \$ 5]$ and [17, Chapters II and III]. Since $\mu_{0}=\inf \{\sigma(T(\pi / 2))\}$ it follows that $m(\cdot, \pi / 2)$ is regular for $\mu=r e(\lambda)<\mu_{0}$, and in particular on $\left(-\infty, \mu_{0}\right)$. From an application of the Dirichlet formula $(2.10 \mathrm{a})$ or $(2.10 \mathrm{~b})$ to the solution $\psi=\theta+m(\cdot, \pi / 2) \phi$ of (1.2) introduced in $[4, \$ 2]$ we obtain

$$
\int_{a}^{\infty}\left[p \psi^{\prime 2}+q \psi^{2}-\mu_{0} \psi^{2}\right]-\left(\mu-\mu_{0}\right) \int_{a}^{\infty} \psi^{2}=m(\mu, \pi / 2) \quad\left(\mu \in\left(-\infty, \mu_{0}\right)\right) .
$$

Now since $\psi \in \Delta$, we may apply inequality (2.13), or (2.14) to prove that the first term on the left-hand side of equation (5.1) is nonnegative; since $\mu<\mu_{0}$ and $\psi$ is not null, it follows that

$$
m(\mu, \pi / 2)>0, \quad \mu \in\left(-\infty, \mu_{0}\right) .
$$

Also from $[4, \$ 10]$ or $[17, \S 3.9]$ it is known that

$$
m(\mu, 0) m(\mu, \pi / 2)=-1, \quad \mu \in\left(-\infty, \mu_{0}\right) .
$$

Since our assumption about $f$ implies that $\mu_{0} \in \mathrm{P} \sigma(T(0)) \cup \operatorname{PC} \sigma(T(0))$, the results given in the theorem in $\$ 5$ of [4] show that there is a positive number $s$ 
such that $i \nu m\left(\mu_{0}+i \nu, 0\right)=-s+o(1)$ as $\nu \rightarrow 0^{+}$. We now apply to the analytic function $\left(\lambda-\mu_{0}\right) m(\lambda, 0)$ the analysis used to prove Theorem 2 of $[7, \S 15]$ to show that $\left(\mu-\mu_{0}\right) m(\mu, 0)=-s+o(1)$ as $\mu \rightarrow \mu_{0}^{-}$. This last result is a contradiction of (5.2) and (5.3) since $s$ is positive, and thus the assumption that $\mu_{0} \epsilon$ $\mathrm{P} \sigma(T(0)) \cup \mathrm{PC} \sigma(T(0))$ is not tenable. This in turn implies that it is not possible for equality to hold in (2.13) or (2.14) for nonnull $f$, when $\mu_{0} \in \operatorname{Co}(T(\pi / 2)$ ).

6. Remarks About Theorem 1. In the regular case we have, for any $f$ in $\mathcal{D}(\pi / 2, \pi / 2)$,

$$
\begin{aligned}
\int_{a}^{b}\left[p f^{\prime 2}+q f^{2}\right] & =\left.p f^{\prime} f\right|_{a} ^{b}+\int_{a}^{b} f M[f]=(T(\pi / 2, \pi / 2) f, f) \\
& \geq \mu_{0}(f, f)=\mu_{0} \int_{a}^{b} f^{2}
\end{aligned}
$$

because the operator $T(\pi / 2, \pi / 2)$ is bounded below. As noted in the introduction, the boundedness of the operator follows from results in the book by Glazman [7].

To extend this inequality to $D$ we require the following analogue of Theorem 3.

Lemma 4. Suppose that the coefficients $p$ and $q$ satisfy the conditions (2.1). Then given a function $f$ in $D$ and $\epsilon>0$, there exists a function $g$ in $\mathcal{D}(\pi / 2, \pi / 2)$ for which

$$
\left|\int_{a}^{b} \not f^{\prime 2}-\int_{a}^{b} p g^{\prime 2}\right|<\epsilon, \quad\left|\int_{a}^{b} f^{2}-\int_{a}^{b} g^{2}\right|<\epsilon, \quad\left|\int_{a}^{b} q f^{2}-\int_{a}^{b} q g^{2}\right|<\epsilon .
$$

Proof. For a given positive number $\eta$ we can choose a continuously differentiable function $\phi$ with the property that $\phi(a)=0=\phi(b)$ and $\int_{a}^{b}\left|f^{\prime}-\phi\right|^{2}<\eta$. For example, take

$$
\phi_{n}(x)=\sqrt{\pi / 2} \sin [n(x-a) \pi /(b-a)] \text { and } \phi=\sum_{n=1}^{N} \alpha_{n} \phi_{n^{*}} .
$$

Then define $g$ by $g(x)=f(a)+\int_{a}^{x} \phi(t) d t$. From this point the proof goes like the proof of Theorem 3, with obvious simplifications.

Lemma 4 is used to extend the inequality (1.1) to $D$ in the same way Theorem 3 was used in the singular case.

We turn now to the case of equality. First, it is clear that equality holds in (1.1) for $f=\alpha \psi_{0^{*}}$. Conversely, if $f$ is a function in $D$ for which equality holds in (1.1), then it follows from classical results that $M[f]=\mu_{\delta} f$, since this equation is the Euler equation for the functional in (1.1). (See Chapter $V$ of the text by Reid [16].) A minimizing function for (1.1) must also satisfy the classical transversality conditions of the calculus of variations, which in this case are $f^{\prime}(a)=0, f^{\prime}(b)=0$. (For a discussion of these conditions see the text by Akhiezer [1].) It follows that $f$ is a multiple of $\psi_{0}$. 
As a consequence of Theorem 1 , we are able to show that $\mu_{0}$ is less than the smallest eigenvalue of either of the operators $T(0, \pi / 2), T(\pi / 2,0)$ or $T(0,0)$. We denote these eigenvalues, respectively, by $\lambda_{1}, \lambda_{2}$, and $\lambda_{3}$, and we denote the corresponding normalized eigenfunctions by $\psi_{1}, \psi_{2}$, and $\psi_{3}$. Since $\psi_{i} \in D, i=$ $1,2,3$, it is clear that $\mu_{0} \leq \lambda_{i}$ because otherwise we would have $\int_{a}^{b}\left[p \psi^{\prime 2}+q \psi_{i}^{2}\right]$ $=\lambda_{i}<\mu_{0}$, which is impossible according to Theorem 1. Moreover, if $\mu_{0}=\lambda_{i}$ for any $i \in\{1,2,3\}$, then $\psi_{i}$ would be a minimizing function for (1.1) and it follows that $\psi_{i}^{\prime}(a)=0=\psi_{i}^{\prime}(b)$. But by definition of $\psi_{i}, \psi_{i}(a)=0$, or $\psi_{i}(b)=0$ and therefore $\psi_{i}(x)=0(x \in[a, b])$, which contradicts the assumption that $\int_{a}^{b} \psi_{i}^{2}=1$.

The fact that $\mu_{0}<\lambda_{i}$ leads us to the interesting observation that is not possible to approximate functions in $D$ with functions in $\mathscr{D}=\mathscr{D}(0, \pi / 2) \cup$ $\mathscr{D}(\pi / 2,0) \cup \mathfrak{D}(0,0)$ in the sense of Lemma 4. Indeed, if Lemma 4 were true with $\mathscr{D}(\pi / 2, \pi / 2)$ replaced by $\mathscr{D}(0, \pi / 2), \mathcal{D}(\pi / 2,0)$ or $\mathcal{D}(0,0)$, then the argument used in the proof of Theorem 1 would yield the result that for some $i$ in $\{1,2,3\}$

$$
\int_{a}^{b}\left[p f^{\prime 2}+q f^{2}\right] \geq \lambda_{i} \int_{a}^{b} f^{2}>\mu_{0} \int_{a}^{b} f^{2}
$$

for all $f$ in $D$, which is known to be false by Theorem 1 .

We summarize these results in

Theorem 5. If the bypotheses of Theorem 1 bold and if $i \in\{1,2,3\}$, then $\mu_{0}<\lambda_{i}$. Moreover, for each function $f$ in $D$ there is an $\epsilon>0$ such that at least one of the following inequalities bolds:

$$
\left|\int_{a}^{b} p f^{\prime 2}-\int_{a}^{b} p g^{\prime 2}\right| \geq \epsilon, \quad\left|\int_{a}^{b} q f^{2}-\int_{a}^{b} q g^{2}\right| \geq \epsilon,
$$

or

$$
\left|\int_{a}^{b} f^{2}-\int_{a}^{b} g^{2}\right| \geq \epsilon
$$

for all $g \in \mathfrak{D}=\mathscr{D}(0, \pi / 2) \cup \mathscr{D}(\pi / 2,0) \cup \mathscr{D}(0,0)$.

Note. There is a similar result to the second part of Theorem 5 in the singular case. If it is known that

$$
\mu_{0}=\inf \{\sigma(T(1 / 2 \pi))\}<\inf \{\sigma(T(0))\}
$$

then the set $\mathscr{D}(0)$ cannot be dense in $D$ in the sense of Theorem 3 .

7. Examples. In this section we consider two applications of the results given in Theorem 2; the first from the bound condition and the second from the integrable condition.

$$
\text { Let } a=1 \text { and for } \tau \in[0, \infty) \text {, }
$$

$$
p(x)=x^{\tau}(x \in[1, \infty)), \quad q(x)=0(x \in[1, \infty)) .
$$


In this case the spectrum of the differential operators $T(\alpha)$ may be found directly from properties of the solutions of the differential equation

$$
-\left(x^{\tau} y^{\prime}\right)^{\prime}=\lambda y \quad \text { on }[1, \infty) \text {. }
$$

Solutions of (7.1) may be obtained explicitly in terms of Bessel functions of order $v= \pm\left|(\tau-1)(\tau-2)^{-1}\right|$ when $\tau \neq 2$, and when $\tau=2$ in terms of elementary functions; see, for example, [ $7 \$ 17$, Example 2]. These results enable us to determine the spectrum as follows (see also $[10, \S 34]$ ):

(i) When $0 \leq \tau<2$

$$
\operatorname{P} \sigma(T(\pi / 2))=\operatorname{PC} \sigma(T(\pi / 2))=\varnothing, \quad \operatorname{C} \sigma(T(\pi / 2))=[0, \infty) ;
$$

i.e. $\mu_{0}=0$.

(ii) When $\tau=2$

$$
\operatorname{Po}(T(\pi / 2))=\operatorname{PC} \sigma(T(\pi / 2))=\varnothing, \quad \operatorname{C} \sigma(T(\pi / 2))=[1 / 4, \infty) ;
$$

i.e. $\mu_{0}=1 / 4$.

(iii) When $2<\tau<\infty$

$$
\operatorname{C} \sigma(T(\pi / 2))=\operatorname{PC} \sigma(T(\pi / 2))=\varnothing, \quad \operatorname{P} \sigma(T(\pi / 2))=\left\{\mu_{t}: t=0,1, \cdots\right\}
$$

where $1 / 4<\mu_{0}<\mu_{1}<\ldots$.

In this case

$$
D=\left\{f \in L^{2}(1, \infty): f \in A C_{10 \mathrm{c}}[1, \infty) \text { and } x^{\tau / 2} f^{\prime} \in L^{2}(1, \infty)\right\}
$$

and we have the following inequalities, all best possible in the sense of Theorem 2:

(i) When $0<\tau<2, \int_{1}^{\infty} x^{\tau} f^{2}>0\left(f \in D, \int_{1}^{\infty} f^{2}=1\right)$.

(ii) When $r=2, \int_{1}^{\infty} x^{2} f^{2}>1 / 4\left(f \in D, \int_{1}^{\infty} f^{2}=1\right)$.

(iii) When $2<\tau<\infty, \int_{1}^{\infty} x^{\tau} f^{\prime 2} \geq \mu_{0}>1 / 4\left(f \in D, \int_{1}^{\infty} t^{2}=1\right)$, with equality in (iii) if and only if $f$ is a multiple of the eigenfunction of (7.1) corresponding to $\mu_{0}$ for $f^{\prime}(1)=0$.

$$
\begin{aligned}
& \text { Let } a=0 \text { and } \\
& p(x)=1(x \in[0, \infty)), \text { for some } r \in[1, \infty), q \in L^{r}(0, \infty) .
\end{aligned}
$$

In this case we can obtain a lower bound for $\inf \{\sigma(T(\pi / 2))\}$ from an extension of the analysis given in the proof of the Theorem of $\$ 7$ in [8]. The method used in [8] to obtain a lower bound for the spectrum of $T(0)$ may be extended to prove the same bound for $T(\pi / 2)$ and so gives the following result

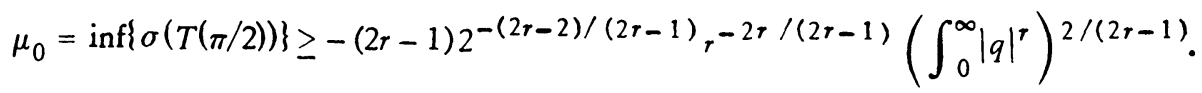

If we substitute this result in (2.14) then we obtain an inequality for $f \in D$ in which only $q$ appears explicitly. Two examples are

(i) if $q \in L(0, \infty)$ then 


$$
\int_{0}^{\infty}\left[f^{\prime 2}+q f^{2}\right] \geq-\left(\int_{0}^{\infty}|q|\right)^{2} \int_{0}^{\infty} f^{2} \quad(f \in D)
$$

(ii) if $q \in L^{2}(0, \infty)$ then

$$
\int_{0}^{\infty}\left[f^{\prime 2}+q f^{2}\right] \geq-\frac{3}{4} \quad\left(\int_{0}^{\infty}|q|^{2}\right)^{4 / 3} \int_{0}^{\infty} f^{2} \quad(f \in D)
$$

where in both examples

$$
D=\left\{f \in L^{2}: f \in A C_{1 \text { oc }}[0, \infty), f^{\prime} \in L^{2}(0, \infty),|q|^{1 / 2} f \in L^{2}(0, \infty)\right\} .
$$

These two examples should be seen in the case when $q$ takes negative values in $[0, \infty)$ or even when $q$ is unbounded below. In both examples we have

$$
\mathrm{C} \sigma(T(\pi / 2)) \cup \operatorname{PC} \sigma(T(\pi / 2))=[0, \infty), \quad \operatorname{P} \sigma(T(\pi / 2)) \subset(-\infty, 0)
$$

and $\mathrm{P} \sigma$ may be empty but can be infinite $w$ ith a limit point at 0 ; if $\mathrm{P} \sigma \neq \varnothing$ then $\mu_{0}<0$ and will be the smallest (isolated) eigenvalue of $T(\pi / 2)$. For the properties of $\sigma(T(\pi / 2))$ in these examples see $[8, \S 1]$ or $[10$, Chapter 2$]$.

\section{REFERENCES}

1. N. I. Ahiezer, The calculus of variations, GITTL, Moscow, 1955; English transl., Blaisdell, Waltham, Mass., 1962. MR 17, 861; MR 25 \#5414.

2. G. A. Bliss, Lectures on the calculus of variations, Univ. of Chicago Press, Chicago, Ill., 1946. MR 8, 212.

3. J. S. Bradley, Adjoint quasi-differential operators of Euler type, Pacific J. Math. 16 (1966), 213-237. MR 34 \#409.

4. J. Chaudhuri and W. N. Evcritt, On the spectrum of ordinary second-order differential operators, Proc. Roy. Soc. Edinburgh A 68 (1968), 95-119.

5. R. Courant and D. Hilbert, Methoden der Mathematischen Physik. Vol. I, Springer, Berlin, 1931; English transl., Interscience, New York, 1953. MR 16, 426.

6. W. N. Everitt, On the limit-point classification of second order differential operators, J. London Math. Soc. 41 (1966), 531-534. MR 34 \#410.

7. - On an extension to an integro-differential inequality of Hardy, Littlewood and Pólya, Proc. Roy. Soc. Edinburgh A 69 (1972), 295-333.

8. - On the spectrum of a second order linear differential equation with a p-integrable coefficient, Appl. Anal. 2 (1972), 143-160.

9. W. N. Everitt, M. Giertz and J. Weidmann, Some remarks on a limit-point and separation criterion for second-order, ordinary differential expressions, Math. Ann. 200 (1973), 335-346.

10. I. M. Glazman, Direct methods of qualitative spectral analysis of singular differential operators, Fizmatgiz, Moscow 1963; English transl., Israel Program for Scientific Translations, Jerusalem, 1965; Davey, New York, 1966. MR 32 \#2938; \#8210.

11. G. H. Hardy, J. E. Littlewood and G. Pólya, Inequalities, Cambridge Univ. Press, New York, 1934.

12. T. Kato, Perturbation the ory for linear operators, Die Grundlehren der math. Wissenschaften, Band 132, Springer-Verlag, New York, 1966. MR $34 \# 3324$. 
13. L. Lichtenstein, Zur Variationrechung, Kgl. Ges. Wiss. Nach. Math.-Phys. Kl. 2 (1919), 161-192.

14. M. A. NaYmark, Linear differential operators. Part II, GITTL, Moscow, 1954; English transl., Ungar, New York, 1968. MR 16, 702; MR 41 \#7485.

15. C. R. Putnam, An application of spectral theory to a singular calculus of variations problem, Amer. J. Math. 70 (1948), 780-803. MR 10, 718.

16. W. T. Reid, Ordinary differential equations, Wiley, New York, 1971. MR $42 \# 7963$.

17. E. C. Titchmarsh, Eigenfunction expansions associated with second-order differential equations. Part I, 2nd ed., Clarendon Press, Oxford, 1962. MR 31 \#426.

18. R. We instock, Calculus of variations, with applications to physics and engineering, McGraw-Hill, New York, 1952. MR 14, 661.

DEPARTMENT OF MATHEMATICS, UNIVERSITY OF TENNESSEE, KNOXVILLE, TENNESSEE 37916

DEPARTMENT OF MATHEMATICS, UNIVERSITY OF DUNDEE, DUNDEE, SCOTLAND 